\title{
Molecular-dynamics simulation of the effect of ions on a liquid-liquid interface for a partially miscible mixture
}

Richard L. Rowley

rowley@byu.edu

Kent E. Wardle

Eric Carlson

Douglas Henderson

Follow this and additional works at: https://scholarsarchive.byu.edu/facpub

Part of the Chemical Engineering Commons

\section{Original Publication Citation}

Wardle, Kent E., Eric Carlson, Douglas Henderson, and Richard L. Rowley. "Molecular-dynamics simulation of the effect of ions on a liquid-liquid interface for a partially miscible mixture." The Journal of Chemical Physics 12 (24): 7681-7688

\section{BYU ScholarsArchive Citation}

Rowley, Richard L.; Wardle, Kent E.; Carlson, Eric; and Henderson, Douglas, "Molecular-dynamics simulation of the effect of ions on a liquid-liquid interface for a partially miscible mixture" (2004). Faculty Publications. 444.

https://scholarsarchive.byu.edu/facpub/444

This Peer-Reviewed Article is brought to you for free and open access by BYU ScholarsArchive. It has been accepted for inclusion in Faculty Publications by an authorized administrator of BYU ScholarsArchive. For more information, please contact ellen_amatangelo@byu.edu. 


\title{
Molecular-dynamics simulation of the effect of ions on a liquid-liquid interface for a partially miscible mixture
}

\author{
Kent E. Wardle, Eric Carlson, ${ }^{\text {a) }}$ Douglas Henderson, ${ }^{\text {a) }}$ and Richard L. Rowley ${ }^{\text {b) }}$ \\ Department of Chemical Engineering, Brigham Young University, Provo, Utah 84602
}

(Received 1 December 2003; accepted 20 January 2004)

\begin{abstract}
Molecular-dynamics simulations were performed to model the effect of added salt ions on the liquid-liquid interface in a partially miscible system. Simulations of the interface between saturated phases of a model 1-hexanol+ water system show a bilayer structure of 1-hexanol molecules at the interface with $-\mathrm{OH}$ heads of the first layer directed into the water phase and the opposite orientation for the second layer. The alignment of the polar $-\mathrm{OH}$ groups at the interface stabilizes a charge separation of sodium and chloride ions when salt is introduced into the aqueous phase, producing an electrical double layer. Chloride ions aggregate nearer the interface and sodium ions move toward the bulk water phase, consistent with the explanation that the $-\mathrm{OH}$ alignment presents a region of partial positive charges to which the hydrated chloride atoms are attracted. Ions near the interface were found to be less solvated than those in the bulk phase. An electric field was also applied to drive ions through the interface. Ions crossing the interface tended to shed water molecules as they entered the hexanol bilayer, leaving a trail of water molecules. Stabilization and facilitated transport of the ion by interactions with the second layer of hexanol molecules appeared to be an important step in the mechanism of sodium ion transport. (C) 2004 American Institute of Physics.
\end{abstract} [DOI: 10.1063/1.1669374]

\section{INTRODUCTION}

Knowledge of the molecular-level behavior of ions in an aqueous solution and at a liquid-liquid interface (LLI) can be an important component in understanding and designing modern chemical processes involving the transport of ions across an interface. A molecular-level understanding of the transport mechanism across a LLI and its relationship to the LLI structure may also provide valuable design capabilities for important biological, pharmacuetical, chemical, and industrial processes in which liquid-liquid extraction is a key step. ${ }^{1,2}$ The influence of salt concentration on the bulk properties and the structure of the LLI is often an important component of these processes, and we report here fundamental studies using molecular-dynamics (MD) simulations on model systems of a LLI in the presence of salt.

Simonin and Hendrawan ${ }^{3}$ performed experimental studies on the effect of salt concentration on the transfer of solutes across a LLI. They found that ion solvation dynamics were key in the ion transfer mechanism. Molecular-dynamics simulations performed by Koneshan et al., ${ }^{4}$ examined the residence time of solvated water molecules in the first coordination shell of various ionic species in order to evaluate ion mobility and to better understand the process by which ions shed solvating water molecules during transfer from an aqueous to a nonaqueous phase. Using MD simulations, Wang $^{5}$ reported a break-up of the hydrogen bonding network near a LLI which may have implications on ion transfer.

\footnotetext{
a) Also at: Department of Chemistry, Brigham Young University, Provo, UT 84602.

b) Author to whom correspondence should be addressed. Electronic mail: rowley@byu.edu
}

Various MD simulation studies of ion transfer dynamics across LLIs were performed by Schweighofer and Benjamin $^{6,7}$ and others ${ }^{8}$ in which the formation of water "fingers" behind the transfer of an ion from the aqueous phase to organic was observed. It was also shown that this effect increased for smaller, more strongly solvated ions for which the energy cost for transfer was higher.

Previous experimental studies of saline solutions near a LLI identified the formation of an electrical double-layer. Volkov et al. ${ }^{9}$ in a review of double-layer theory asserted that understanding the formation of this double-layer is vital to explaining the mechanism of ion transfer across the interface. The MD simulations by Jungwirth and Tobias ${ }^{10}$ showed the presence of the double-layer at an air-water interface. They determined that chloride ions remain nearer to the interface due to their larger size and weaker solvation. To our knowledge, this has not been observed in simulations of the LLI, nor has an adequate explanation for the apparent charge separation at the interface been given. MD simulations offer the opportunity to observe any such charge separation in model saline LLI systems and proffer a molecular explanation for the charge separation.

In a previous study, we simulated the LLI for the partially miscible system water+1-hexanol. ${ }^{11}$ The LLI for that system was found to contain a bilayer of hexanol molecules. Starting from the water side of the interface, the 1-hexanol interaction sites were preferentially ordered as $\mathrm{H}, \mathrm{O}, \alpha-\mathrm{CH} 2$, $\beta-\mathrm{CH} 2, \gamma-\mathrm{CH}_{2} \cdots \mathrm{CH}_{3}$ in the first layer of 1-hexanol molecules, followed by the reverse pattern for the second layer. The purpose of this MD study is to further elucidate the molecular-level structure of a model water/hexanol LLI with added $\mathrm{NaCl}$. We report here a charge separation in the aque- 


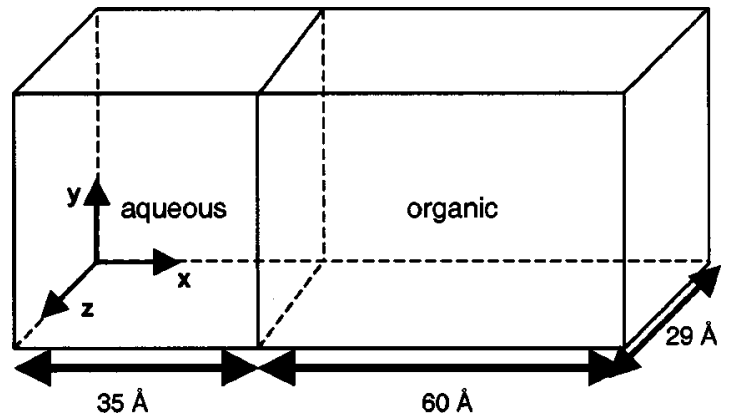

FIG. 1. Simulation cell dimensions.

ous phase that results from the structuring of the hexanol molecules at the LLI, consistent with experimentally reported occurences of an electrical double layer at an LLI. We also examine ion solvation characteristics and differences between solvation in the bulk phase, at the LLI, and as the ion passes through the interface in response to an applied field.

\section{SIMULATION METHODOLOGY}

NVT MD simulations were performed using 956 water molecules, 144 hexanol molecules, and 18 sodium and 18 chloride ions $(\sim 1.1 \mathrm{M})$ for a total of 1236 molecules per simulation cell. All of the simulations were performed at 298 $\mathrm{K}$ and at an overall density approximately corresponding to ambient pressure. Figure 1 shows a diagram of our simulation cell. The techniques for simulating the LLI are the same that were used by us in previous simulations of a model water $+n$-hexane system and a model water +1 -hexanol system. ${ }^{11}$ Details of the simulations are available in those previous studies, and we report here a summary of the method with specific details given only for changes or new methods associated with the addition of the ions. Periodic boundary conditions were used in all three directions so that the system is modeled as repeating images (in the $x$ direction) of infinite slabs (in the $y$ and $z$ directions) of alternating aqueous and organic bulk phases. As in our previous study, ${ }^{11}$ the length of the organic phase was extended, by the choice of how many molecules of each kind were used, to better accommodate the structuring of the hexanol molecules that occurs due to the presence of the interface and better approximate a bulk phase at the center of the organic slabs. Time steps were 2.5 fs and a fourth-order-correct predictorcorrector integrator was used to move the system forward in time. The particle-particle-particle mesh (P3M) method of the Ewald sum was used to handle the long-range interactions.

As shown in our previous study, ${ }^{11}$ the resultant LLI was stable and equilibrated after 100000 time steps. Simulations initiated from different starting configurations produced similar bulk phases and LLI structure after 100000 time steps with the exception of the amount of water soluble in the 1-hexanol phase. 1-Hexanol was found to be insoluble in the water phase consistent with the negligible experimental solubility. Although the experimental solubility of the water in 1-hexanol is $\sim 30 \mathrm{~mol} \%$, previous osmotic MD simulations for the models used here determined it to be $\sim 35$ mol\%. ${ }^{11}$ The simulations reported here were initiated at a mole fraction of $30 \mathrm{~mol} \%$ water in the organic phase, but generally equilibrated at $\sim 23 \mathrm{~mol} \%$ water in the organic phase. Accurate determination of the equilibrium solubility was not the intent of this study, but a decrease in water solubility in the organic phase due to the presence of ions in the aqueous phase is to be expected.

Water was modeled with the extended simple point charge (SPCE) model ${ }^{12}$ and 1-hexanol was modeled with a united atom model in which interactions with the $-\mathrm{CH}_{x}$ groups were treated with a single Lennard-Jones (LJ) site. The hydroxyl $\mathrm{O}$ and $\mathrm{H}$ interactions were modeled as the sum of a LJ interaction and a coulombic interaction. The latter interaction was based on a partial positive charge assigned at the nuclear center of the $\mathrm{H}$ atom and an equal but negative charge assigned at the oxygen center. These partial charge values were obtained from Jorgensen. ${ }^{13}$ Bond lengths and angles were held rigid at their equilibrium values using distance constraints while applying Gaussian mechanics. ${ }^{14}$ Internal conformational changes in hexanol were governed by a torsional potential, $u_{\text {tors }}$, of the Ryckaert-Bellemans form $^{15}$

$$
\frac{u_{\mathrm{tors}}(\varphi)}{k}=\sum_{i=0}^{5} a_{i} \cos ^{i}(\varphi),
$$

where $k$ is Boltzmann's constant and $\varphi$ is the dihedral angle. The coefficients in Eq. (1) for rotation about $\mathrm{C}-\mathrm{C}$ bonds were obtained directly from the literature ${ }^{15}$ while those for rotation about the $\mathrm{C}-\mathrm{O}$ bond were regressed from a model potential developed by Jorgensen. ${ }^{16}$ The model parameters and the coefficients $a_{i}$ are given in Table I. Sodium and chloride ions were modeled with appropriate integer electronic charges of plus and minus one, respectively, located at the centers of the ions, coupled with the LJ potential previously used by others. ${ }^{17}$

As mentioned, the equilibrated water-1-hexanol interface was independent of the starting configuration of the water and 1-hexanol molecules and so this equilibrium configuration was used as the starting point for simulations involving sodium and chloride ions. Because the LLI presents a rather large energy barrier for ion transfer between phases, we began one set of simulations with all ions in the aqueous phase and another set with all the ions in the organic phase. Equilibrated system configurations and results were averaged over $\sim 2.5 \mathrm{~ns}$ in the case of the aqueous-initiated ions and $7.75 \mathrm{~ns}$ for the less-stable organic-initiated ion simulations. The equilibrium bulk densities and mutual solubilities of the two phases are in agreement with experimental data and expected behavior. No 1-hexanol molecules appeared in the aqueous phase, even with no added salt, consistent with the experimental solubility of $0.12 \mathrm{~mol} \%$. As mentioned above, the equilibrated solubility of water in 1-hexanol was $\sim 23 \mathrm{~mol} \%$ with ions present in the aqueous phase, consistent with the expectation that added salt would lower the solubility from the experimental value of $30 \mathrm{~mol} \%$.

In order to view the ion transport mechanism and the solvation behavior of ions as they cross the interface, we also conducted a pair of simulations in which applied electric fields of 0.316 and $0.421 \mathrm{~V} / \AA$ were used to drive individual 
TABLE I. Model parameters used in the simulations.

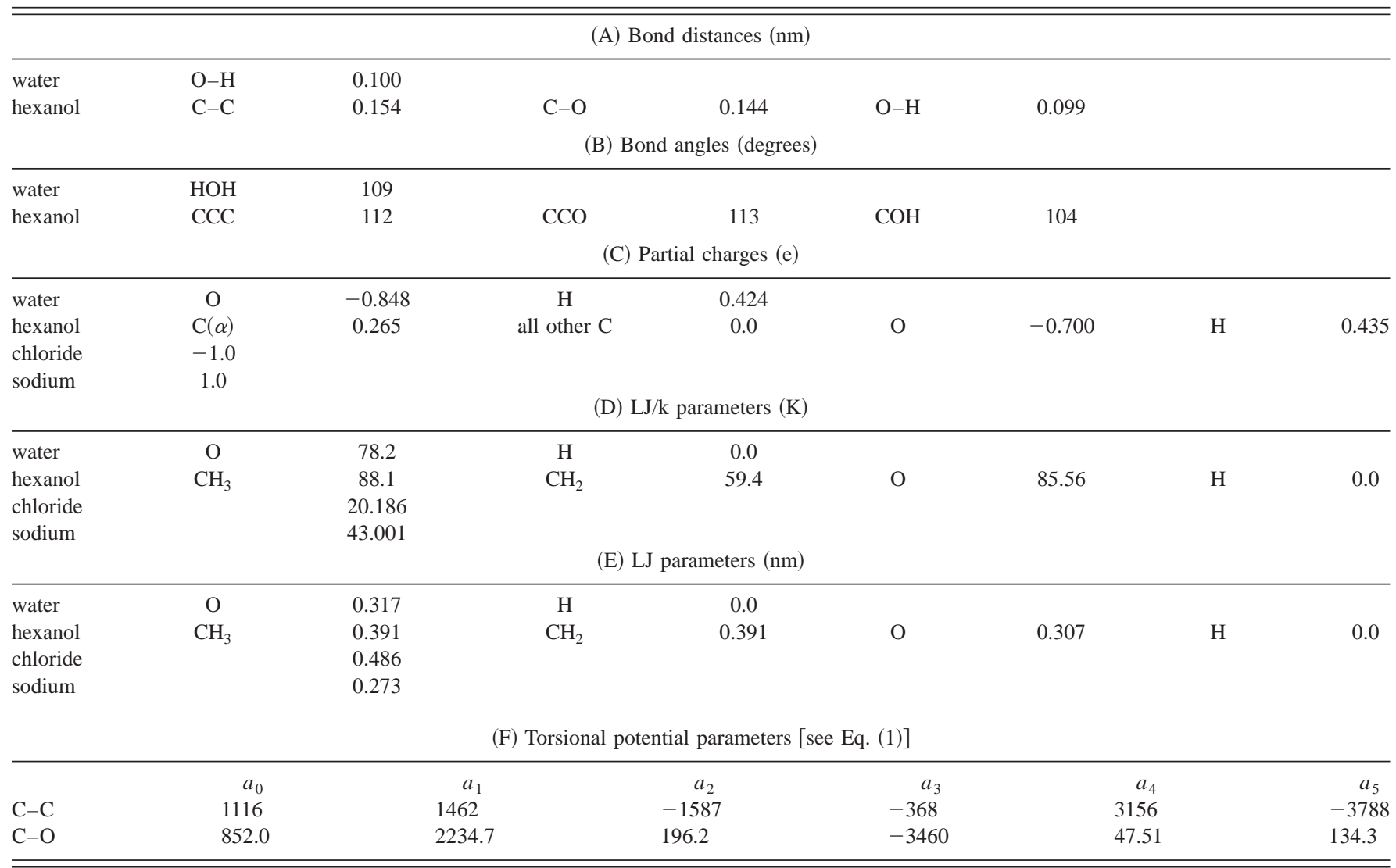

sodium and chloride ions through the interface. This was not meant to model the system under the effect of an applied field, but rather to investigate the structural changes as a single ion moves through the LLI. Therefore, the driving field was applied only to a single ion of each type at a time.

\section{RESULTS AND DISCUSSION}

\section{A. Effect of ions on the LLI}

Figure 2 shows the density profile for the simulation with 18 pairs of salt ions. As observed in our previous study, ${ }^{11}$ there is a highly structured bilayer of 1 -hexanol molecules that forms in the organic phase. Figure 2 shows a very

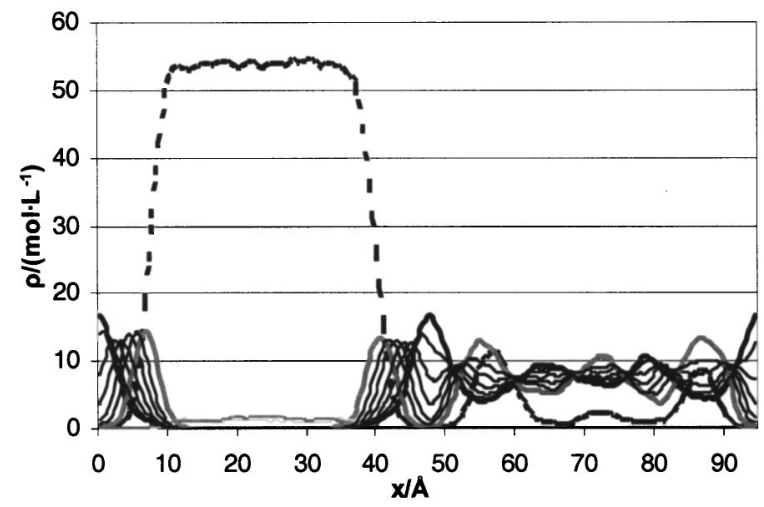

FIG. 2. Density histogram of water $\mathrm{O}(---),-\mathrm{CH}_{3}(-),-\mathrm{CH}_{2}$ (centered unbroken line), alcohol $\mathrm{H}$ (grey -$), \mathrm{Na}^{+}(-----)$, and $\mathrm{Cl}^{-}$ $(-)$ sites as a function of simulation cell length. pronounced and distinct ordering of the 1-hexanol interaction sites in the order $\mathrm{H}, \mathrm{O}, \alpha-\mathrm{CH}_{2}, \beta-\mathrm{CH}_{2}, \gamma-\mathrm{CH}_{2}$, etc., sequentially to the $-\mathrm{CH}_{3}$ group. This peak is approximately twice the size of the peaks for the other groups because the second portion of the bilayer begins with a reverse ordering of this second group of 1-hexanol molecules from the overlapping $-\mathrm{CH}_{3}$ group down the alkyl chain to the hydroxyl $\mathrm{O}$ and finally to the hydroxyl $\mathrm{H}$ group. The addition of the salt to the organic phase evidently has little effect upon this bilayer structure which we also observed in our previous studies of the neat hexanol-water interface. The water dissolved in the organic phase is, as before, observed to cluster in the $-\mathrm{OH}$ cages created by the reverse micellar-like orga-

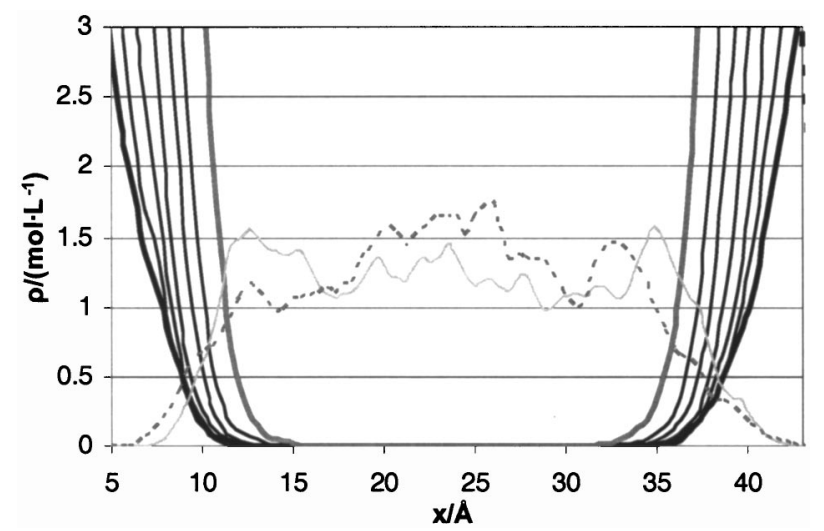

FIG. 3. Expanded density histogram of the aqueous phase showing the $\mathrm{Na}^{+}$ (-----), and $\mathrm{Cl}^{-}$(grey - ) distribution (See Fig. 2 for key). 


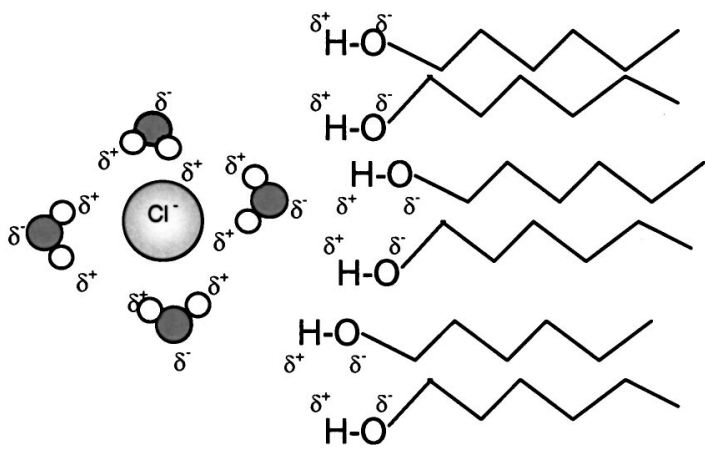

FIG. 4. Interaction of solvated $\mathrm{Cl}^{-}$complex with interface.

nization of the hexanol molecules in the "bulk" organic phase. The small size of our organic phase and consequently the presence of the interfacial bilayer structure of hexanol molecules renders these water-dissolving structures relatively static in time. Time averages in larger simulations should show a uniform average water density in the bulk phase rather than the static positions of the dissolved water seen in the time average density plot for our smaller system shown in Fig. 2.

Figure 3 shows an enlargement of the time-averaged histogram for just the aqueous phase. We observe a higher number density for chloride ions near the interface and a higher density for the sodium ions in the middle of the water phase. This charge separation or electric double layer was observed in all of our simulations. Although our observation of enhanced $\mathrm{Cl}^{-}$ion density at the LLI is similar to that of Jungwirth and Tobias ${ }^{10}$ at a vapor-liquid interface, there are additional interactions in the case of the LLI studied here that produce these results. In addition to the difference in solvation due to ion size, the structure of the LLI between water and hexanol plays a large role in the observed ion separation. As can be seen in Figs. 2 and 3, the rather dominant alignment of the hexanol molecules at the interface with the positively charged hydrogen on the alcohol group extending toward the aqueous phase, stabilized by the hydrogen bonding with water in the aqueous phase, presents a plane of positive

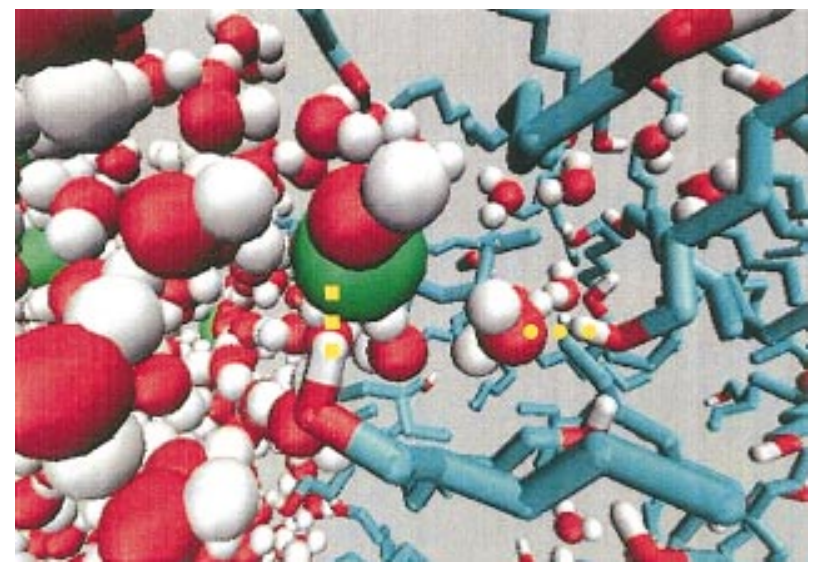

FIG. 5. (Color) Snapshot of solvated $\mathrm{Cl}^{-}$ion (green) in the interface showing direct $\mathrm{H}_{\text {alcohol }}$ to $\mathrm{Cl}^{-}$(口, yellow) and solvated $\mathrm{H}_{\text {alcohol }}$ to $\mathrm{Cl}\left(\mathrm{H}_{2} \mathrm{O}\right)_{x}^{-}$ (००, yellow) interactions.

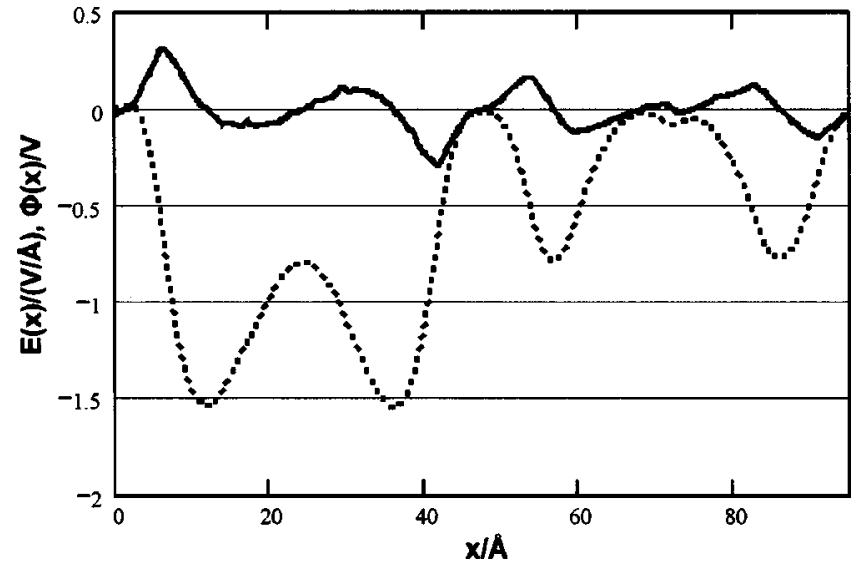

FIG. 6. Electric field (continuous) and electrostatic potential (dashed) as a function of location throughout the cell. The interface locations are at $\sim 6$ and $\sim 42 \AA$.

charge to which the negatively charged solvated chloride ions are attracted as sketched in Fig. 4. Figure 5 shows a snapshot ${ }^{18}$ of the LLI where the higher density of $\mathrm{Cl}^{-}$ions at the interface can be seen as well as a visual suggestion of the solvated chlorides interacting with the hydroxyl $\mathrm{H}$ of the first hexanol layer at the interface. This attraction includes direct interaction with the $\mathrm{Cl}^{-}$ion within the first solvation shell and with the solvated complex.

The electric potential, $\Phi$, and electric field, $E$, were calculated as a function of cell position throughout the cell by integrating over the charge distribution using Poisson's equation from the LLI outward to the middle of the bulk fluid. Arbitrary boundary conditions at of $\Phi=0.0$ at $x / L=0$ and 1.0 were used in the integration, so the potential obtained is relative to the values at the LLI. The time average charge density distribution was first determined at 200 equidistant points across the cell. This charge density distribution was then fitted to a 100-term Fourier series. This permitted an analytical integration of Poisson's equation to obtain the electrostatic field and potential. The results are shown in Fig. 6. Note the bimodal shape of the electrostatic potential in the aqueous portion of the cell. The symmetric potential well indicates the attraction of negative ions to both LLI's on

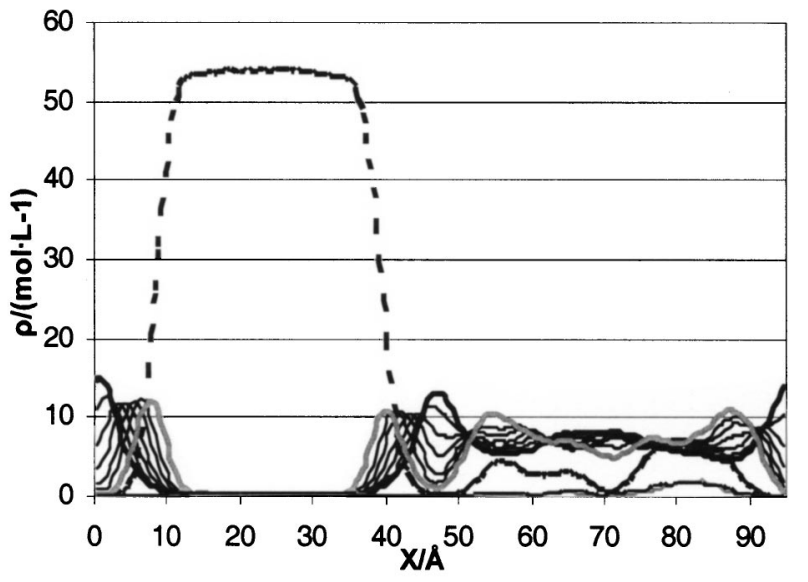

FIG. 7. Density profile of equilibrated organic-initiated system showing ions in water pockets within the organic phase (See Fig. 2 for key). 


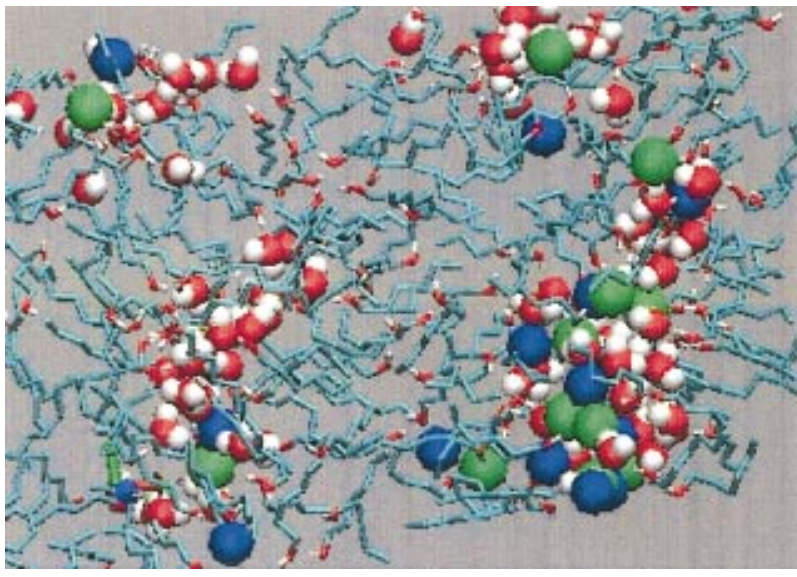

FIG. 8. (Color) Equilibrated ion $\left(\mathrm{Cl}^{-}\right.$, green) $\left(\mathrm{Na}^{+}\right.$, blue) clusters with water in $\mathrm{OH}$-cages in organic phase when the ions were initially placed in the organic phase.

either side of the aqueous phase. The relative potential hill in the middle shows the presence of a higher density of positive charges. The extent of our water phase is yet too small to achieve a true bulk fluid in the center of the aqueous phase as we do not obtain in this simulation a flat potential region in the center of the aqueous phase.

\section{B. lons in the organic phase}

It was unclear whether the simulations were ergodic because there is such a high energy barrier for transfer of an ion across the interface into the organic phase. The barrier of course does not preclude the stability of ions in the organic phase; it only diminishes the rate of occurrence below that for which our simulations of $3.2 \times 10^{6}$ time steps (7.75 ns) are capable of observation. We, therefore, repeated the simulations, but this time started the simulations with all of the ions randomly distributed in the organic phase. Figure 7 shows a density profile for the second run of this system. Only 5 ions of each species, maintaining electroneutrality, crossed the LLI into the aqueous phase; all other ions remained in the water-filled $\mathrm{OH}$ cages of the alcohol shown in Fig. 8. The ions in these water-filled cages were less solvated by water molecules than in the aqueous phase and tended to

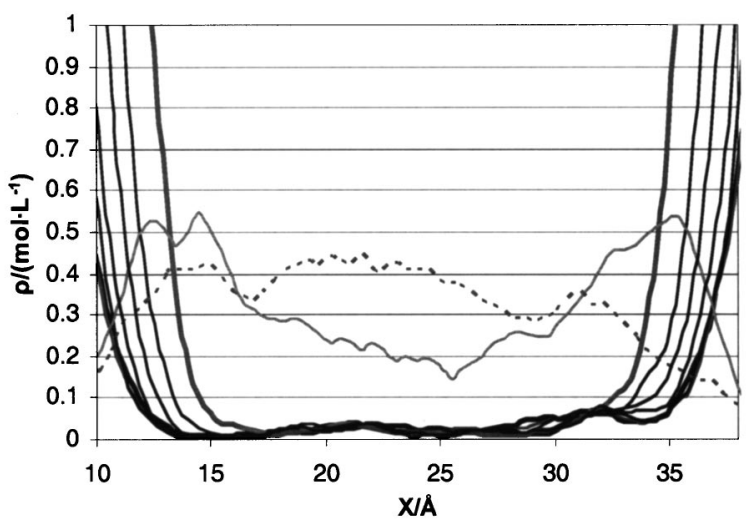

FIG. 9. Density profile of aqueous phase of organic-initiated system showing the electric double layer with $\mathrm{Cl}^{-}$near the interface (See Fig. 2 for key).

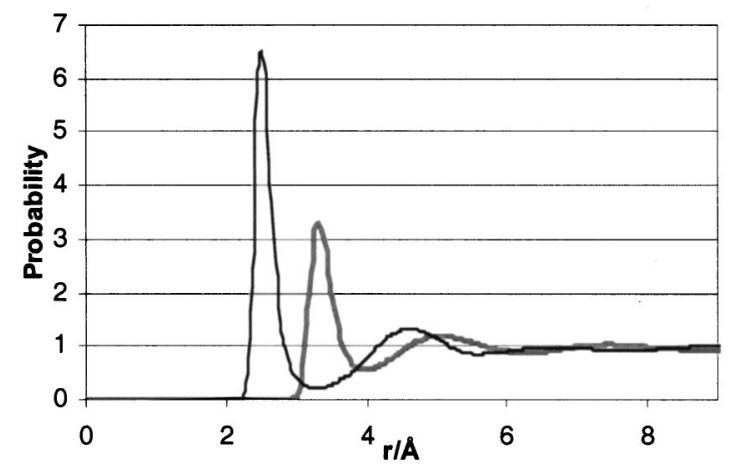

FIG. 10. Radial Distribution Function for $\mathrm{Na}^{+}(-)$, and $\mathrm{Cl}^{-}$(grey - ) to water $\mathrm{O}$

ion pair. The electrical double layer was again evident in the aqueous phase, even at the much more dilute concentration of only 5 ions of each species. Figure 9 shows the evident double-layer in the density profile for the aqueous region of this system for one of the two replicate simulations initiated in this manner.

\section{Ion solvation}

The $\mathrm{Na}-\mathrm{O}$ and $\mathrm{Cl}-\mathrm{O}$ radial distribution functions were calculated from the simulations in the bulk aqueous phase and the results are shown in Fig. 10. A cut-off distance for the solvent first coordination shell was taken to be the minimum beyond the first maximum in the radial distribution function. Thus, all water molecules with an ion-O distance less than this cut-off value were viewed as part of the first solvation shell for the ion. This solvation cut-off distance was found to be $3.35 \AA$ for sodium and $3.9 \AA$ for chloride ions.

In the bulk phase, an average of 6 water molecules solvate the sodium ions and 7 water molecules solvate the chloride ions. However, it was evident from visualization of the simulations that less solvation occurs in the LLI region. Solvation profiles were, therefore, obtained as a function of cell position in the same manner as described in the preceding paragraph. In order to view the interaction of ions with alcohol groups at the interface, we also determined the number of

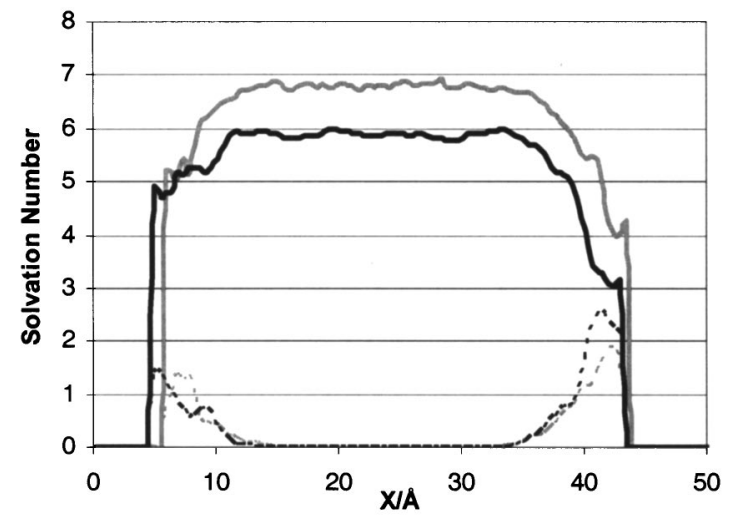

FIG. 11. Solvation profile across the aqueous phase showing $\mathrm{Na}^{+}$to water $\mathrm{O}(-), \mathrm{Na}^{+}$to hexanol $\mathrm{O}(---), \mathrm{Cl}^{-}$to water $\mathrm{H}$ (grey - ), and $\mathrm{Cl}^{-}$to hexanol H (grey - - ) coordinations within the respective cutoffs. 


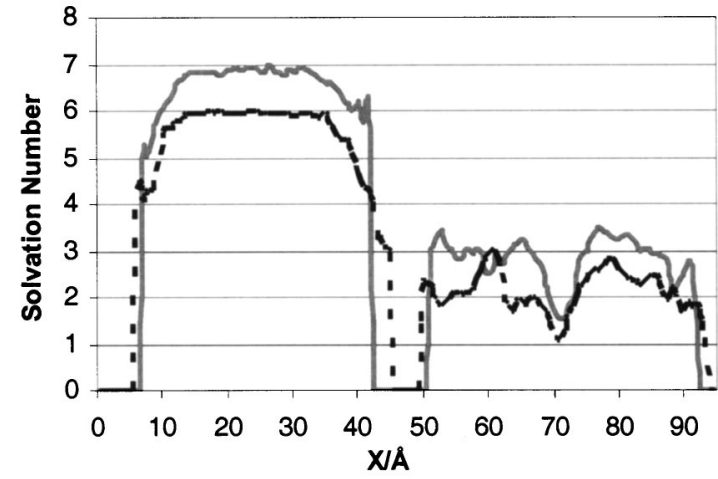

FIG. 12. Solvation profile across the entire cell of the organic-initiated system showing the number of solvating waters for $\mathrm{Cl}^{-}(-)$, and $\mathrm{Na}^{+}$ (- - -) in the organic phase is approximately half that of ions in the aqueous phase.

alcohol groups $(\mathrm{Na}-\mathrm{OH}$ and $\mathrm{Cl}-\mathrm{HO})$ within the solvation cut-off distance. These results are shown in Fig. 11. It also appeared that this de-solvation was more dramatic for the sodium ions than for the chloride ions. As noted from previous studies, the break-up of the hydrogen bonding network in the water molecules near the interface causes the water molecules to be less stable nearer the interface. As solvated ions approach the interface, the water molecules' attraction to them is not sufficient to overcome the negative effects of the interface and fewer water molecules are able solvate. Similar results were obtained for both runs of the system in which the ions were all started in the organic side of the simulation cell. At equilibrium, the majority of the ions remained in pockets of water in the organic phase (see Fig. 8). Such ions were solvated with less than half as many water molecules as those in the aqueous phase (see Fig. 12). Ions are stablized in the organic phase by solvation with the water located in the hydrogen-bonded cages of the alcohol and by pairing with their counterions.

\section{Transport of ions across the interface}

By driving single ions through the interface with an electric field applied to a specific ion, we were able to view the solvation dynamics as the ion passes through the interface.
The solvation profiles, including the interaction with water and hexanol molecules for both the sodium and chloride ions, are shown in Figs. 13(a) and 13(b) for an applied field of $0.316 \mathrm{~V} / \AA$. As the ions approach and cross the interface and the organic bilayer, water molecules are increasingly likely to be stripped from the transferring ion as they proceed into the organic bilayer. This was the case for ions of both types. Similar results were seen for the larger applied field of $0.421 \mathrm{~V} / \mathrm{A}$. It also appeared that the chloride ion moved more freely than the smaller sodium ion. The motion of the sodium ion was particularly slowed by the hydrophobic tails in the center of the bilayer. Water molecules from the solvation sphere appeared to trail the ions as they crossed the interface and entered the hydrophobic region of overlapping organic tails. This dragging of water by the ion is strongly reminiscent of, but more attenuated than, the "water fingers" observed by others ${ }^{6-8}$ as ions were transferred under the influence of an applied field.

Figure 13 and animated views of the ion transport process suggest that sodium ion transfer through the bilayer is a facilitated process. The interactions between hexanol oxygen atoms and the sodium ion appear to be essential in overcoming the energy barrier for transference through the organic bilayer. In Fig. 13(a) hexanol-O interactions are seen to replace the water-O interactions with the moving sodium ion in its solvation shell. The interaction with hexanol-O atoms reaches a maximum near the center (hydrophobic region) of the bilayer. These interactions appear to stabilize and facilitate the transport of the sodium ion across the interface and hydrophobic bilayer region. Snapshots illustrating the progress of a transferring sodium ion with an applied field of 0.421 V/A are shown in Figs. 14(a)-14(d). Figures 14(a)14(c) are equally incremented in time while the time interval between Figs. 14(c) and 14(d) is much longer. Interfacial hexanol molecules on the aqueous side of the bilayer (highlighted in Fig. 14) rotate and follow the ion while some of the hexanol molecules in the organic side of the bilayer (also highlighted in Fig. 14) turn about and stabilize the ion while it is in the hydrophobic region of the bilayer. These interactions can be visualized in Fig. 14(b) where a hexanol molecule that had been pointing into the interface has turned about to follow and apparently stabilize the sodium within the hydrophobic bilayer region. The sodium ion stalls in this
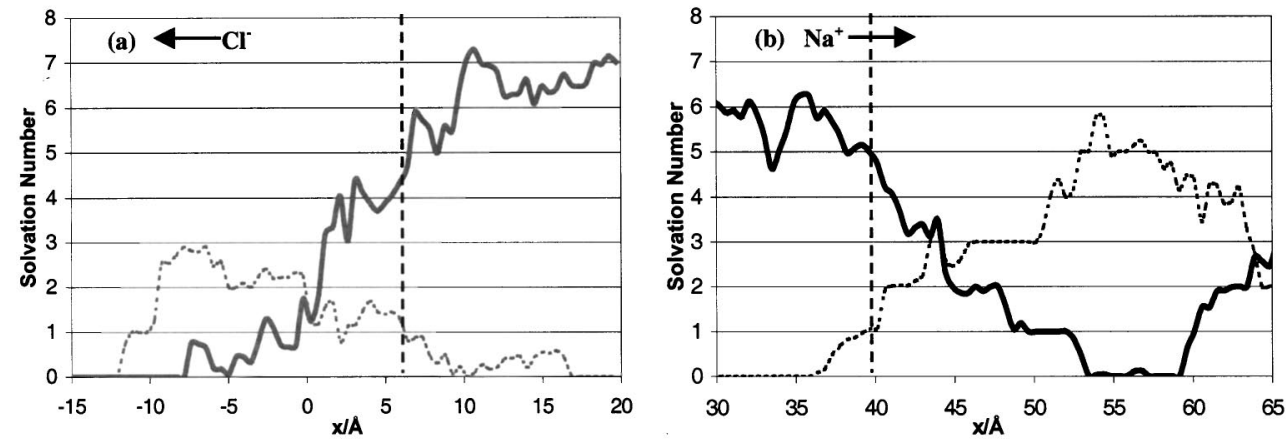

FIG. 13. Solvation profile for the electric field (0.316 V/Å) driven transport of (a) $\mathrm{Na}^{+}$and (b) $\mathrm{Cl}^{-}$across LLI (See Fig. 11 for key). Arrows show direction of counterion movement and the dashed vertical lines represent the approximate interface location. 

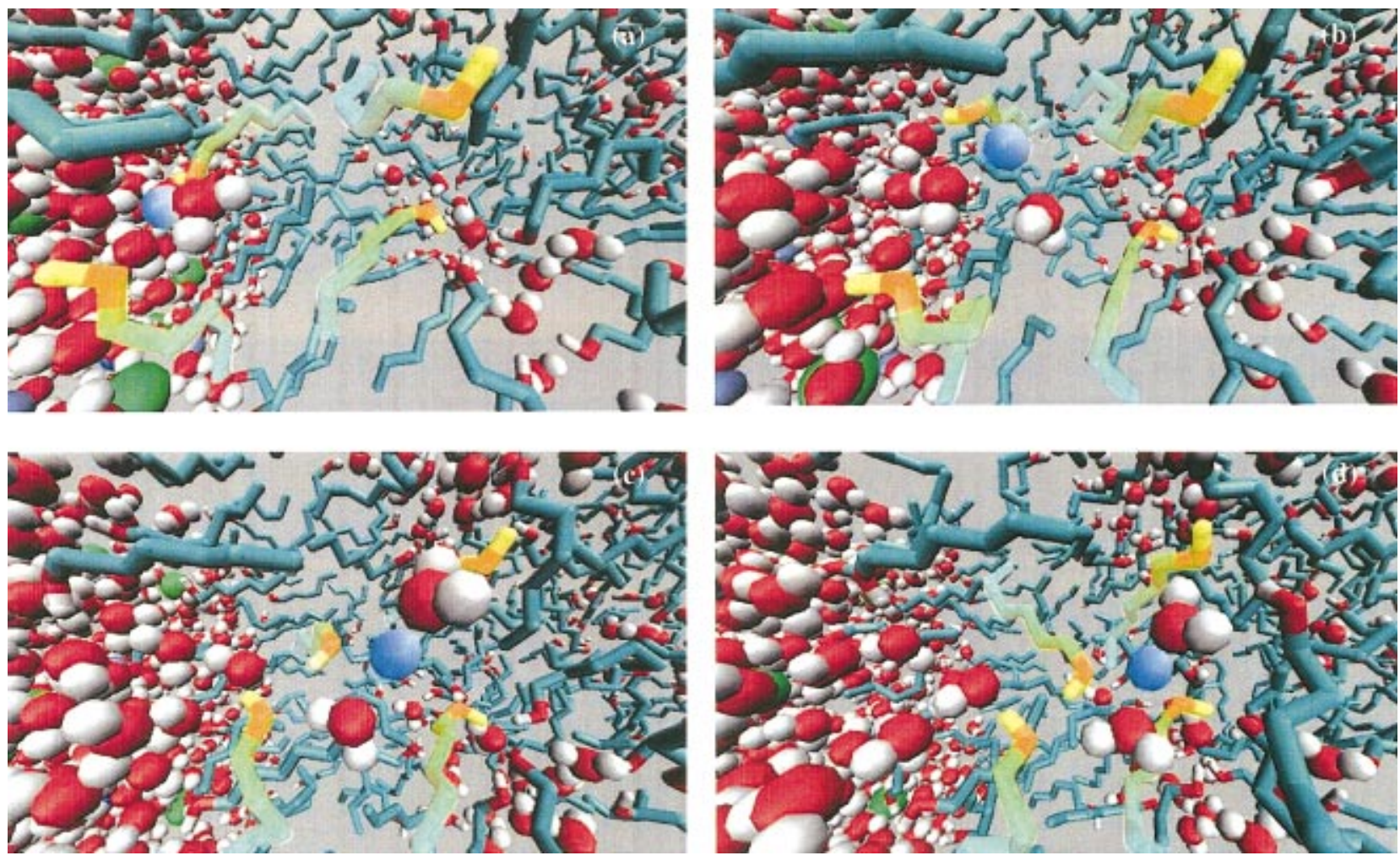

FIG. 14. (Color) Sequential snapshots (a)-(d) of forced transfer of $\mathrm{Na}^{+}$ion. (a) $\mathrm{Na}^{+}$ion begins to cross interface. (b) Ion brings solvating water molecules and is followed by two hexanol molecules (highlighted with yellow tint, on left). (c) Ion slows in hydrophobic bilayer region while hexanol molecules (highlighted, on right) from the organic side of the bilayer reorient to stabilize the ion. (d) Hexanol molecules from the organic side of the bilayer along with the two from the aqueous side surround the ion and facilitate its transport.

region until a critical number of stabilizing hexanol-O $(\sim 5$ and 6) solvate it and accompany it through the bilayer [see Fig. 14(d)]. Facilitated transport of this type did not appear to be as important for the larger chloride ion.

\section{CONCLUSIONS AND SUMMARY}

The MD simulations performed in this study have described the behavior of aqueous $\mathrm{Na}^{+}$and $\mathrm{Cl}^{-}$ions relative to a hexanol-water interface. The presence of an electrical double layer was observed, consistent with previous experimental inferences. The attraction of solvated chloride ions to the plane of the partially positive $\mathrm{H}$ atoms in the highly structured hexanol bilayer is a plausible explanation for the observed enhanced chloride ion density near the interface and the depleted sodium ion density in the same region.

It was also apparent from the simulations that as ions approach the interface, they tend to be less solvated than in the bulk phase. This de-solvation appears to be due to increased interactions with the hexanol $\mathrm{OH}$ heads, coupled with the increasing instability of water molecules nearer the interface. A balance of de-solvation coupled with the stabilizing effect of the surrounding hexanol molecules appears to be a key step in the movement of an ion across the interface from the aqueous to the organic phase. This is particularly true for the sodium ion.

A simulation of the double layer formed at a liquid/ liquid interface that models the resulting capacitance is of interest. This would require a larger computational cell than that used here, but should be feasible. There have been few, if any, simulations of the capacitance at a liquid-liquid interface. However, there has been some theoretical interest ${ }^{19}$ indicating that the capacitance at the liquid-liquid double layer should be enhanced over that of an electrolyteelectrode interface.

\section{ACKNOWLEDGMENT}

Acknowledgment is made to the donors of the Petroleum Research Fund, administered by the American Chemical Society, for support of this project.

${ }^{1}$ A. Pfennig and A. Schwerin, Ind. Eng. Chem. Res. 37, 3180 (1998).

${ }^{2}$ M. Lauterbach, E. Engler, N. Muzet, L. Troxler, and G. Wipff, J. Phys. Chem. B 102, 245 (1998).

${ }^{3}$ J. P. Simonin and H. Hendrawan, J. Phys. Chem. B 104, 7163 (2000).

${ }^{4}$ S. Koneshan, J. C. Rasaiah, R. M. Lynden-Bell, and S. H. Lee, J. Phys. Chem. B 102, 4193 (1998).

${ }^{5}$ H. B. Wang, Masters Thesis, Dept. of Chemical Engineering, Brigham Young University (2003).

${ }^{6}$ K. J. Schweighofer and I. Benjamin, J. Phys. Chem. 99, 9974 (1995).

${ }^{7}$ K. Schweighofer and I. Benjamin, J. Phys. Chem. A 103, 10274 (1999).

${ }^{8}$ M. Lauterbach, E. Engler, N. Muzet, L. Troxler, and G. Wipff, J. Phys. Chem. B 102, 245 (1998)

${ }^{9}$ A. G. Volkov, D. W. Deamer, D. L. Tanelian, and V. S. Martin, Prog. Surf. Sci. 53, 1 (1996).

${ }^{10}$ P. Jungwirth and D. J. Tobias, J. Phys. Chem. B 104, 7702 (2000).

${ }^{11}$ H. B. Wang, E. Carlson, D. Henderson, and R. L. Rowley, Mol. Simul. 29, 777 (2003).

${ }^{12}$ H. J. C. Berendsen, J. R. Grigera, and T. P. Straatsma, J. Phys. Chem. 91, 6269 (1987). 
${ }^{13}$ W. L. Jorgensen, J. Am. Chem. Soc. 103, 335 (1981).

${ }^{14}$ D. J. Evans, W. G. Hoover, B. H. Failor, B. Moran, and A. J. C. Ladd, Phys. Rev. A 28, 1016 (1983).

${ }^{15}$ J. P. Ryckaert and A. Bellemans, Chem. Phys. Lett. 30, 123 (1975).

${ }^{16}$ W. L. Jorgensen, J. Phys. Chem. 90, 1276 (1986).
${ }^{17}$ K. Schweighofer and I. Benjamin, J. Chem. Phys. 112, 1474 (2000).

${ }^{18}$ Graphic molecular renderings were made with VMD software. W. Humphrey, A. Dalke, and K. Schulten, J. Mol. Graphics 14, 33 (1996).

${ }^{19}$ C. M. Pereira, W. Schmickler, A. F. Silva, and M. J. Sousa, Chem. Phys. Lett. 268, 13 (1997). 